\title{
Analysis of Ultraviolet Absorption Lines in NGC 6543 and NGC 6826
}

\author{
M. Bremer ${ }^{1}$, M. Grewing ${ }^{1}$, J. Barnstedt ${ }^{2}$ and N. Kappelmann ${ }^{2}$ \\ ${ }^{1}$ Institute de Radio Astronomie Millimétrique (IRAM), Domaine \\ Universitaire de Grenoble, 300 rue de la Piscine, 38406 St Martin \\ d'Hères, France ${ }^{2}$ Institut für Astronomie und Astrophysik, Universität \\ Tübingen, Auf dem Sand 1, 72076 Tübingen, Germany
}

\begin{abstract}
During the ORFEUS-SPAS II mission (November 19th December 7th 1996, aboard the space shuttle Columbia), the elliptical planetary nebulae NGC 6543 and NGC 6826 have been studied with the Echelle spectrometer. The instrumental wavelength range and resolution are $900 \AA-1400 \AA$ and $0.1 \AA$, respectively $(R=10000)$, which provides detailed information about emission and absorption lines which are inaccessible for ground-based observations. We present a new analysis of the absorption lines imposed on the spectra of the central stars.
\end{abstract}

\section{Introduction}

NGC 6543 and NGC 6826 are two "classical" PNe which have been studied extensively from radio to X-ray wavelengths. NGC 6543 is known to have a complex multiple shell structure with significant variations in excitation, from neutral (e.g. O I) to highly ionized species (P V, O VI, S VI). Molecular hydrogen absorption lines in the ORFEUS II spectra indicate that $\mathrm{H}_{2}$ is present along the line of sight, but the question remains if this component is associated with the nebula or intervening Galactic gas. NGC 6543's radial velocity of $-65.7 \mathrm{~km} / \mathrm{s}$ makes it a prime candidate to distinguish between Galactic and nebula matter by line shift, but a problem arises from the uncertain absolute velocity zero of the ORFEUS II Echelle spectra.

In the progress report, we focus on $\mathrm{H}_{2}$ transitions and atomic/ionic species found at the same velocity. We have identified a number of $\mathrm{H}_{2}$ transitions (25 for NGC 6543,55 for NGC 6826) and constructed curves of growth in order to determine the $\mathrm{H}_{2}$ column density.

\section{Data analysis}

The calibrated ORFEUS II Echelle spectra (Barnstedt et al. 1999, A\&AS 134, 561 ) for NGC 6543 and NGC 6826 were averaged weighted with their integration times, and then analysed with the AIT program packages ECH and WFIT.

The selected $\mathrm{H}_{2}$ transitions for the curve of growth fits are exclusively lines which are not blended with neighboring transitions or ionized lines, which limited the choice considerably. 
As the zero point of the wavelength scale of the spectra contains an unknown offset, we have used the $\mathrm{H}_{2}$ lines as a reference and looked for atomic and ionic species at the same velocity shift.
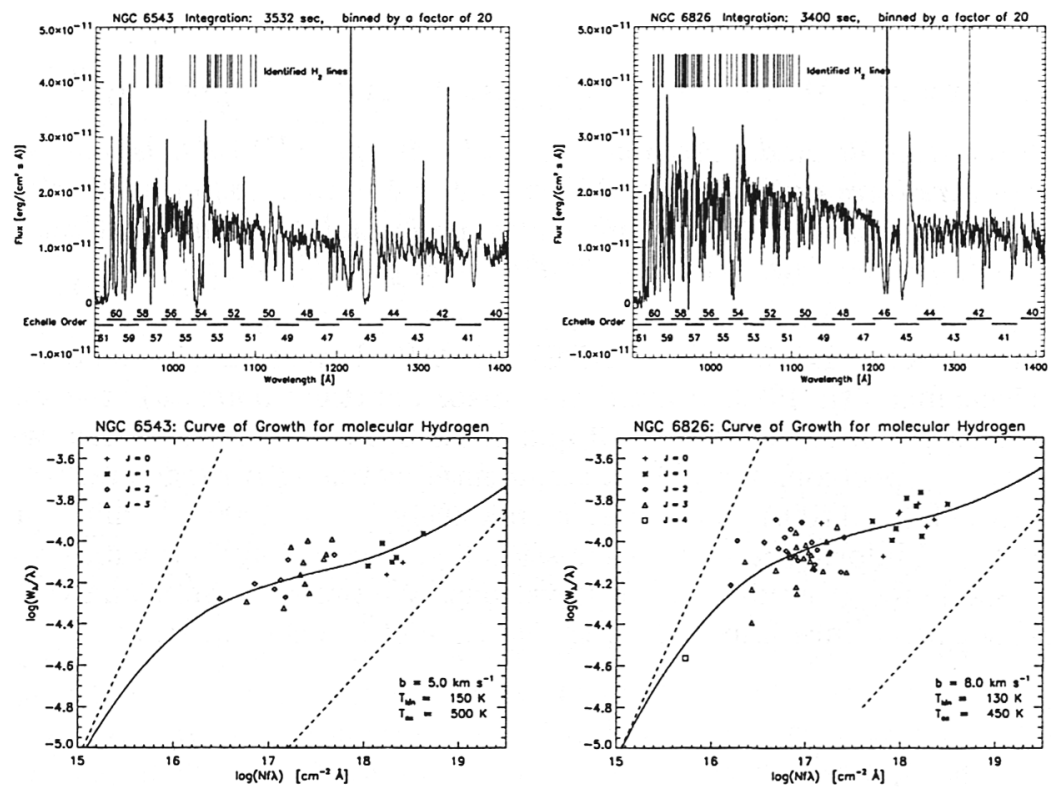

Figure 1. Top row: binned ORFEUS II spectra, with the corresponding curves of growth beneath. Instrumental and terrestrial lines: Test pulse peak $1317 \AA$, airglow 1302, 1305, 1306 and $989 \AA$, geo-corona Lyman $\alpha$ and $\beta$ at 1216 and $1026 \AA$, respectively.

\section{Results}

For NGC 6543, we derive an average velocity shift relative of $\mathrm{H}_{2}$ to the unknown "zero" in the Echelle spectra of $-26.1 \pm 9.1 \mathrm{~km} / \mathrm{s}$, based on 25 lines. For a variety of high and low ionized species (a total of 27 lines), we obtain $-25.7 \pm$ $15.6 \mathrm{~km} / \mathrm{s}$, which matches the $\mathrm{H}_{2}$ values closely. More ionized lines exist, but their identification was not as clear. This indicates that the $\mathrm{H}_{2}$ is associated with the nebula.

The curve of growth analyses of both nebulae indicate that $\mathrm{H}_{2}$ and turbulent velocity are less than a factor of two different, and that there are only minor differences in excitation temperature and kinetical temperature. Assuming a correlation between nebula dust emission and the $\mathrm{H}_{2}$ column density, NGC 6543 should have the larger amount of dust and consequently show a larger amount of reddening. This seems to be confirmed by the extinction measurements. We also note that the kinetic temperatures determined from the curves of growth seem to agree with the dust temperatures determined from IRAS fluxes.

\begin{tabular}{l||c|c|c|l} 
& $\log \left(N_{2}\right)$ & $b\left(\mathrm{~km} \mathrm{~s}^{-1}\right)$ & $\mathrm{T}_{k i n}(\mathrm{~K})$ & $T_{e x}(\mathrm{~K})$ \\
\hline NGC 6543 & 16.85 & 5 & 150 & 500 \\
NGC 6826 & 16.63 & 8 & 130 & 450
\end{tabular}

